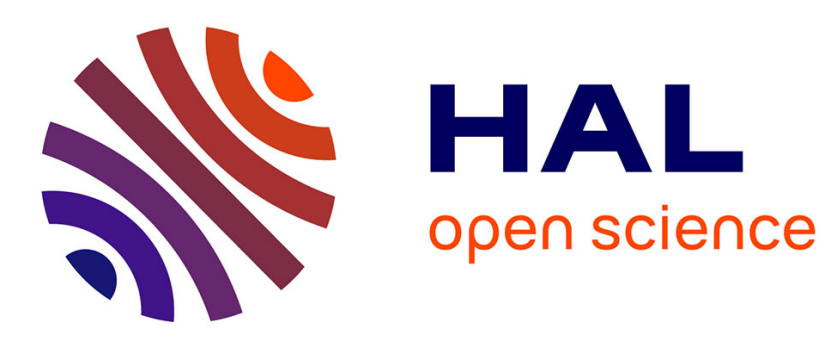

\title{
Black-light continuum generation in a silica-core photonic crystal fiber
}

T. Sylvestre, A. R. Ragueh, M.W. Lee, B. Stiller, G. Fanjoux, B. Barviau, Arnaud Mussot, Alexandre Kudlinski

\section{- To cite this version:}

T. Sylvestre, A. R. Ragueh, M.W. Lee, B. Stiller, G. Fanjoux, et al.. Black-light continuum generation in a silica-core photonic crystal fiber. Optics Letters, 2012, 37, pp.130-132. 10.1364/OL.37.000130 . hal-00708085

\section{HAL Id: hal-00708085 \\ https://hal.science/hal-00708085}

Submitted on 3 May 2021

HAL is a multi-disciplinary open access archive for the deposit and dissemination of scientific research documents, whether they are published or not. The documents may come from teaching and research institutions in France or abroad, or from public or private research centers.
L'archive ouverte pluridisciplinaire HAL, est destinée au dépôt et à la diffusion de documents scientifiques de niveau recherche, publiés ou non, émanant des établissements d'enseignement et de recherche français ou étrangers, des laboratoires publics ou privés. 


\title{
Black-light continuum generation in a silica-core photonic crystal fiber
}

\author{
T. Sylvestre, ${ }^{1, *}$ A. R. Ragueh, ${ }^{1}$ M. W. Lee, ${ }^{1}$ B. Stiller, ${ }^{1}$ G. Fanjoux, ${ }^{1}$ B. Barviau, ${ }^{2}$ A. Mussot, ${ }^{2}$ and A. Kudlinski ${ }^{2}$ \\ ${ }^{1}$ Institut FEMTO-ST, Département d'Optique P. M. Duffieux, Université de Franche-Comté, CNRS, Besançon, France \\ ${ }^{2}$ Laboratoire de Physique des Lasers, Atomes et Molécules, UMR-CNRS 8523 IRCICA, \\ Université des Sciences et Technologies de Lille, Villeneuve d'Ascq, France \\ ${ }^{*}$ Corresponding author: thibaut.sylvestre@univ-fcomte.fr
}

\begin{abstract}
We report the observation of a broadband continuum spanning from 350 to $470 \mathrm{~nm}$ in the black-light region of the electromagnetic spectrum as a result of picosecond pumping a solid-core silica photonic crystal fiber at $355 \mathrm{~nm}$. This was achieved despite strong absorption and a large normal dispersion of silica glass in the UV. Further investigations reveal that the continuum generation results from the interplay of intermodally phase-matched fourwave mixing and cascaded Raman scattering. We also discuss the main limitations in terms of bandwidth and power due to temporal walk-off, fiber absorption, and the photo darkening effect, and we suggest simple solutions.
\end{abstract}

Over the last few years, there have been a number of important efforts to extend the blue and UV sides of supercontinuum (SC) generation in silica-core photonic crystal fibers (PCFs) [트. $\underline{6}$. These efforts are motivated by applications of broadband UV light in biomedical sciences and spectroscopy. While most of the experiments have been performed by pumping in near-IR (NIR) or visible regions, it is remarkable that the use of laser emitting in the UV as a pump for SC generation in PCF has not yet been endeavored. Generation of UV broadband light has, however, recently been reported in a single-mode high-OH fiber [7].

Herein, we demonstrate that despite both the strong absorption and large normal dispersion of silica in the UV, a broadband continuum can be efficiently generated in the black-light region of the electromagnetic spectrum by pumping a PCF with a picosecond microchip laser at $355 \mathrm{~nm}$. The PCF is a standard solid-core silica PCF made by IRCICA. It is similar to those usually used for SC generation when pumping at $1064 \mathrm{~nm}$ in the small anomalous dispersion regime []. It has a triangular lattice with a pitch and hole diameter of 3.88 and $2.73 \mu \mathrm{m}$, respectively. Its cross-sectional image is depicted in Fig. 1(a) together with its dispersion curve for the fundamental mode around $355 \mathrm{~nm}$. At this wavelength, our PCF exhibits a large normal group-velocity dispersion (GVD) dominated by material dispersion. In addition, the PCF carries several propagation modes. Three main transverse modes have been identified numerically using a finite element method. These modes are depicted in Figs. 1(b) and 1(c) together with their inverse group velocities and dispersion curves, respectively. The nonlinear coefficient is $\gamma=34 \mathrm{~W}^{-1} \mathrm{~km}^{-1}$ for the fundamental mode, and its zero-dispersion wavelength (ZDW) is $1044 \mathrm{~nm}$. The fiber loss at $355 \mathrm{~nm}$ was measured as $0.5 \mathrm{dBm}^{-1}$. As a pump laser, we used a passively $Q$-switched frequency-tripled Nd:YAG laser (Teemphotonics Powerchip Series) emitting 300 ps pulses (FWHM) at a repetition rate of $1 \mathrm{kHz}$ and an average power of $22 \mathrm{~mW}$. The output was coupled into our PCF by a $10 \times$ microscope objective. The output spectra were recorded using an optical spectrum analyzer, and the continuum formation was analyzed by means of a variable attenuator. We also used a grating spectrometer $(2400 \mathrm{l} / \mathrm{mm})$ and a CCD color camera for modal and spectral analysis. Figure 2 shows the output spectra after $30 \mathrm{~m}$ of fiber for an increasing coupled pump power ranging from 0.5 to $4 \mathrm{~mW}$. We can clearly see the continuum generation that starts from the pump wavelength and broadens up to the blue. The widest continuum spans $120 \mathrm{~nm}$, from 350 to $470 \mathrm{~nm}$, with an output power of $120 \mu \mathrm{W}$. This corresponds to a low spectral power density of only $1 \mu \mathrm{W} / \mathrm{nm}$. The image in the inset shows the optical mode output of the widest continuum recorded by the color camera. The UV light appears as white, whereas the visible light is in true color. This image shows the continuum is mainly carried by the $\mathrm{LP}_{31}$ mode. Figure 3 details the beginning of the continuum
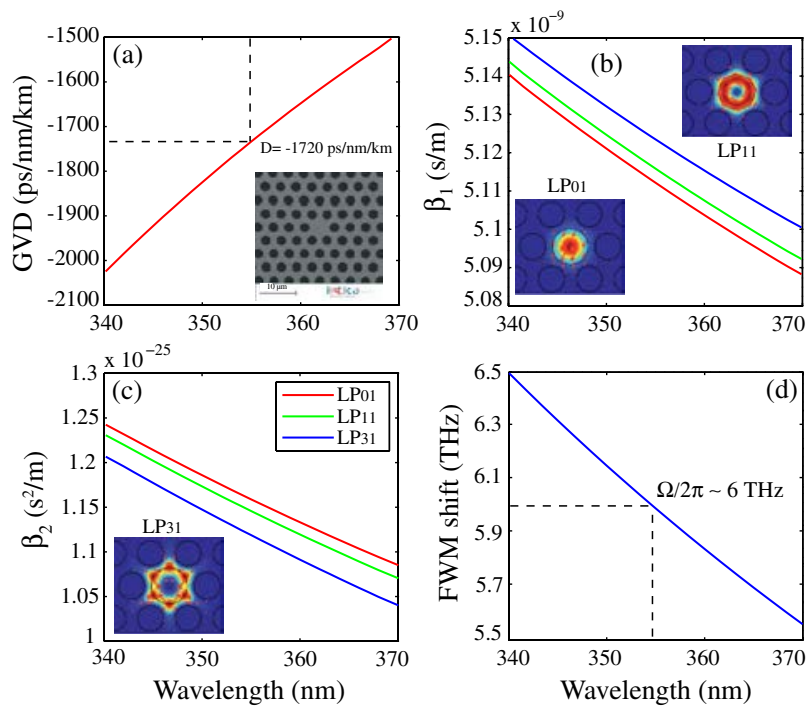

Fig. 1. (Color online) (a) Chromatic dispersion of the fundamental mode versus wavelength. Inset: SEM image of the solid-core PCF. (b), (c) The inverse group velocities and the chromatic dispersions of the three $\mathrm{LP}_{01}$ (red), $\mathrm{LP}_{11}$ (green), and $\mathrm{LP}_{31}$ (blue) modes, respectively, shown in the insets. (d) FWM frequency shift versus wavelength. (See Media 1.) 


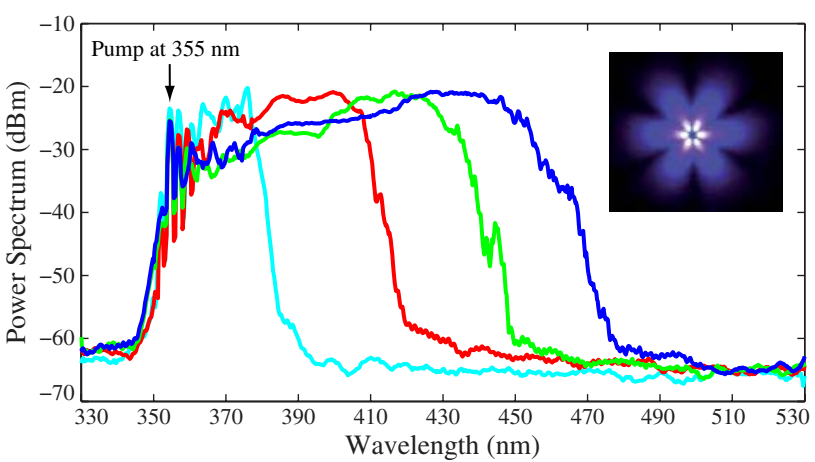

Fig. 2. (Color online) Output spectra recorded at the end of the $30 \mathrm{~m}$ long PCF for a variable coupled pump power from 0.5 to $4 \mathrm{~mW}$. The spectral resolution is $0.5 \mathrm{~nm}$. The inset shows the optical mode output of the blue continuum.

generation by cutting the fiber back to 1 and $4 \mathrm{~m}$, respectively, keeping the same injection conditions. As can be seen in Fig. 3(a), we identify a preliminary intermodal four-wave mixing (FWM) process involving the pump (labeled P) in a mixed-mode distribution (see top image), an anti-Stokes sideband $\left(\mathrm{AS}_{1}\right)$ in the $\mathrm{LP}_{01}$ mode, and a Stokes one $\left(\mathrm{S}_{1}\right)$ in the $\mathrm{LP}_{11}$ mode. The phase-matching condition for this modal FWM process reads as $\kappa=$ $\left(\beta_{1}^{11}-\beta_{1}^{01}\right) \Omega+\beta_{2} \Omega^{2}+2 \gamma P$ [9] , where the first, second, and third terms represent the contributions of modal and chromatic dispersion and nonlinearity, respectively, to the phase mismatch. $\frac{\Omega}{2 \pi}$ is the FWM frequency shift. $\beta_{1}^{11}$ and $\beta_{1}^{01}$ are the inverse group velocities of the $\mathrm{LP}_{11}$ and $\mathrm{LP}_{01}$ modes. $\beta_{2}$ is the GVD coefficient at pump wavelength. Figure 1(d) shows the FWM frequency shift versus the pump wavelength calculated from the above equation. At $355 \mathrm{~nm}$, we found a frequency shift of $6 \mathrm{THz}$, in good agreement with the measurement of Fig. 3(a). We have also checked that all other possible FWM processes involving other modes are not phase-matched. For Fig. 3(b), the pump power exceeds the Raman threshold, which yields two strong first- and second-order Raman Stokes bands, $R_{1}$ and $R_{2}$. Simultaneously, parametric Stokes band $S_{1}$ acts as a second pump and generates both harmonic sideband $S_{2}$ and its own first-order Raman Stokes $\left(\mathrm{SR}_{1}\right)$. Surprisingly, we also observe the efficient generation of two anti-Stokes bands, $\mathrm{AR}_{2}$ and $\mathrm{ASR}_{2}$, far from the pump. We have checked that these waves result from FWM involving six waves, namely the pump $\mathrm{P}$, the parametric band $S_{1}$, and Raman Stokes waves $\mathrm{SR}_{1}$ and second-order $\mathrm{R}_{2}$. In particular, $\mathrm{AR}_{2}$ at $344 \mathrm{~nm}$ has been identified as being second-order Raman anti-Stokes
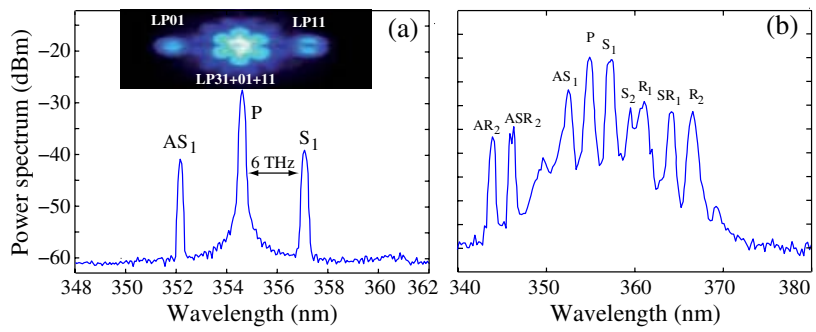

Fig. 3. (Color online) Output spectra recorded at the end of the photonic crystal fiber for shorter fiber lengths : (a) $L=1 \mathrm{~m}$, (b) $L=4 \mathrm{~m}$. The inset shows the modal distribution of FWM.

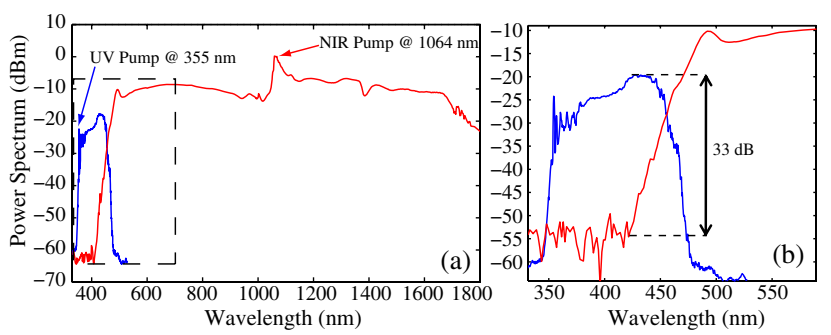

Fig. 4. (Color online) Direct comparison of spectra recorded at the output of the PCF under UV (355 nm, blue) and IR (1064 nm, red) laser pumping. Pump powers are 4 and $25 \mathrm{~mW}$, respectively. (b) A zoom of the part of (a) shown in the dashed box.

upshifted by $26 \mathrm{THz}$ from the pump. To our knowledge, this is the first time that second-order anti-Stokes was efficiently generated in the absence of first-order [10]. We have indeed verified that theses waves are phasematched in higher-order modes of the PCF and fall far from the Raman absorption band. At higher pump power, all Raman and parametric bands together merge and, because of the large Raman gain bandwidth, the CRS evolves toward a smooth continuum, as those shown in Fig. 2. This spectral broadening dynamics is analogous to the one previously reported using visible $532 \mathrm{~nm}$ pump pulses propagating in a dispersion-shifted fiber [9]. Note also that all the anti-Stokes waves reported in Fig. 3 no longer appear in Fig. 2 because of absorption. Moreover, all Raman Stokes bands are mainly carried by the $\mathrm{LP}_{31}$ mode, which explains the optical mode distribution of Fig. 2.

Figure 4 compares the UV continuum generation with that generated by an IR subnanosecond microchip laser at $1064 \mathrm{~nm}$,close to the ZDW of the PCF [8]. The experiments have been performed by replacing the UV laser by the IR one, keeping the same PCF, but with different coupled pump power. As can be seen, the SC generation
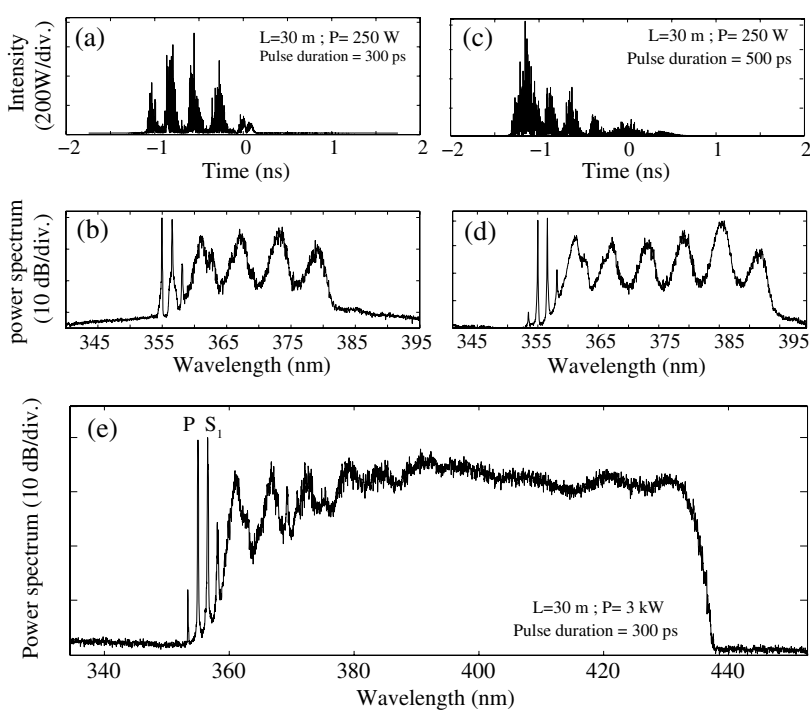

Fig. 5. Numerical simulations of cascaded Raman generation for two input pulse durations and UV continuum generation in the PCF. (a), (c) Temporal and (b),(d) spectral outputs. Parameters are indicated in the figures and text. (e) The UV-visible continuum generation for a peak power of $3 \mathrm{~kW}$. 
using the IR pump spans more than two octaves from $450 \mathrm{~nm}$ to more than $1800 \mathrm{~nm}$, whereas the UV continuum is limited to a third of an octave only. However, the latter covers a new UV-visible spectral window that is not accessible with the IR pump.

Let us now discuss the main limitations. The most important ones are fiber absorption and the photodarkening effect caused by UV radiation. We have indeed observed that the continuum bandwidth strongly decreases after about 20 min due to solarization. These detrimental effects could be mitigated by using specialty high-OH or solarization-resistant optical fiber, as in Ref. [7]. The third drawback is the large normal dispersion of silica that induces a strong pulse walk-off among the Raman pulses and thus limits the continuum extension toward longer wavelengths. This can be easily overcome by using longer nanosecond pulses. To verify the impact of walk-off, we performed split-step numerical simulations based on the generalized nonlinear Schrödinger equation, including the full Raman susceptibility and the wavelength-dependent absorption. As initial conditions, we consider a picosecond pump pulse at $355 \mathrm{~nm}$ together with the parametric band $\mathrm{S}_{1}$ with half of the power to mimic the FWM of Fig. 3. Including the multimode behavior and the FWM intermodal coupling in the numerics is actually very time-consuming and computationally intensive. We also used the one-photon-per-mode model as an input condition. Figures 5(a)-(d) show the results of our numerical computations for $30 \mathrm{~m}$ of singlemode nonlinear propagation and for two pulse durations. As can be seen in Fig. 5(d), for a 500 ps pulse duration, a sixth-order Raman cascade is generated, whereas only four Raman orders occur for a 300 ps pulse duration [Fig. 5(b)]. Figures 5(a) and 5(c) show the corresponding temporal intensities. In both traces, we can clearly identify a train of noiselike Raman pulses that are optically delayed by walk-off. The temporal walk-off is about $300 \mathrm{ps}$, which is close to the pump pulse duration. Consequently, the Raman pulses do not overlap at all after $30 \mathrm{~m}$ of propagation, thus limiting the Raman cascade, as shown in Figs. 5(b) and 5(d). For comparison, we have analytically calculated the Raman pulse walk-off from the following expression: $T=D . L . \Delta \lambda$, where $\Delta \lambda$ is the Raman shift in wavelength. We found a pulse walk-off of 288 ps for $L=30 \mathrm{~m}$, in good agreement with numerical simulations. Finally, the numerical simulation of Fig. $\underline{5(\mathrm{e})}$ was realized with the same conditions but at a higher peak power of $3 \mathrm{~kW}$. It shows that the CRS evolves into a continuum that spans from 355 to about $430 \mathrm{~nm}$, in rather good agreement with the experimental one shown in green in Fig. 2.

In conclusion, we reported the observation of a broadband black-light continuum in a solid-core silica photonic crystal fiber as a result of pumping at $355 \mathrm{~nm}$. The continuum generation has been identified as resulting from the interplay between intermodal FWM and cascaded Raman generation. This fiber-format broadband IR-free black-light source could find possible applications as a Wood's lamp for multicolor excitation of fluorescent proteins in medicine, dermatology, molecular biology, or gas absorption spectroscopy $[7,11]$. Further progress will be pursued in the near future to improve the continuum bandwidth and spectral power density by using solarization-resistant silica optical fibers.

\section{References}

1. J. H. V. Price, T. M. Monro, K. Furusawa, W. Belardi, J. C. Baggett, S. Coyle, C. Netti, J. J. Baumberg, R. Paschotta, and D. J. Richardson, Appl. Phys. B 77, 291 (2003).

2. A. Efimov, A. J. Taylor, F. J. Omenetto, J. C. Knight, W. Wadsworth, and P. S. J. Russell, Opt. Express 11, 910 (2003).

3. G. Genty, M. Lehtonen, and H. Ludvigsen, Opt. Lett. 30, 756 (2005).

4. A. Kudlinski, A. K. George, J. C. Knight, J. C. Travers, A. B. Rulkov, S. V. Popov, and J. R. Taylor, Opt. Express 14, 5715 (2006)

5. J. M. Stone and J. C. Knight, Opt. Express 16, 2670 (2008).

6. S. P. Stark, A. Podlipensky, N. Y. Joly, and P. St. J. Russell, J. Opt. Soc. Am. B 27, 592 (2010).

7. R. J. Bartula, J. W. Walewski, and S. T. Sanders, Appl. Phys. B 84, 395 (2006).

8. W. J. Wadsworth, N. Joly, J. C. Knight, T. A. Birks, F. Biancalana, and P. St. J. Russell, Opt. Express 12, 299 (2004).

9. A. Mussot, T. Sylvestre, L. Provino, and H. Maillotte, Opt. Lett. 28, 1820 (2003).

10. D. A. Akimov, E. E. Serebryannikov, A. M. Zheltikov, M. Schmitt, R. Maksimenka, W. Kiefer, K. V. Dukel'skii, V. S. Shevandin, and Yu. N. Kondrat'ev, Opt. Lett. 28, 1948 (2003).

11. B.-W. Liu, M. Hu, S.-J. Wang, L. Chai, C. Wang, N. Dai, J.-Y. Li, and A. M. Zheltikov, Opt. Lett. 35, 3958 (2010). 